\title{
Tremor, FXTAS and Klinefelter Syndrome
}

Mehanna $\mathbf{R}^{1^{*}}$ and Itin $\mathbf{I}^{2}$

${ }^{1}$ University of Texas Health Science Center, Houston Texas, USA

${ }^{2}$ Center for Neurological Restoration, Cleveland Clinic, Ohio, USA

"Corresponding author: Raja Mehanna, Assistant Professor, Department of neurology, University of Texas Health Science Center at Houston, 6410 Fannin Street Suite 1014, Houston, Texas 77030, USA, Tel: 832.325.7080, Fax:713.512.2239; E-mail: raja.mehanna@uth.tmc.edu

Rec date: Feb 22, 2014, Acc date: May 06, 2014, Pub date: May 09, 2014

Copyright: ( 2014 Mehanna R, et al. This is an open-access article distributed under the terms of the Creative Commons Attribution License, which permits unrestricted use, distribution, and reproduction in any medium, provided the original author and source are credited.

\begin{abstract}
Patients with Klinefelter syndrome have a higher prevalence of postural and action tremor that the general population, but are typically diagnosed before developing tremor. We here report a 68 year old patient diagnosed with Klinefelter syndrome while being tested for FXTAS and review the available literature on this subject.
\end{abstract}

Keywords: Tremor; FXTAS; Klinefelter

\section{Introduction}

Klinefelter syndrome (KS) is the most common aneuploidy in men, affecting $0.1-0.2 \%$ of the general population [1]. While postural and action tremor are more frequent in KS patients than in the general population [2], these patients are generally already diagnosed with KS by the time tremor develops. Indeed, KS is not currently in the clinical list for differential diagnosis of tremor. We here report the case of a 68 year old man diagnosed with $\mathrm{KS}$ while being investigated for fragile $\mathrm{X}$ associated tremor and ataxia syndrome (FXTAS), and review the available literature on this subject.

\section{Case}

A 68 year old right-handed Italian man presented to our clinic for a six years history of bilateral hand action tremor worse on the left. The tremor in his left hand was severe enough to prevent holding a glass or a bottle. He denied any tremor in his head, voice or feet as well as any rest tremor. He also denied softening of his voice, slowness of movement, difficulty with fine motor skills, stiffness or change in his gait. In addition, he denied cognitive problems except for anomia.

He had a past medical history remarkable for diabetes mellitus complicated with neuropathy, chronic kidney disease, obstructive sleep apnea, hyperlipidemia, hypertension and surgical treatment of cataract. The patient was also infertile, and a work up in 1968 diagnosed him with hypospermia.

His medication intake included valsartan, metformin, glyburide, rosuvastatin and gabapentin $600 \mathrm{mg}$ twice a day for painful diabetic neuropathy but with only slight improvement of his tremor. He also was recently started on metoprolol $75 \mathrm{mg} /$ day for his tremor, with good benefit. 80.

His family history was positive only for tremor in his mother at age

The patient denied smoking or drinking alcohol. He had retired from running a sandblasting business, but had dropped out of school in the 7 th grade because of learning difficulty.
On examination, he scored $23 / 30$ on the Montreal Cognitive Assessment (MOCA), missing all recall questions, and losing two additional points for attention and one for fluency. He did benefit from an additional point for having completed less than 12 years of education. On $600 \mathrm{mg}$ twice a day of gabapentin and $75 \mathrm{mg}$ daily on long acting metoprolol, he had a bilateral hand kinetic tremor, worse on the left, without any head, voice, leg or rest tremor. His tremor rating scale was 23, with inability to draw Archimedes spirals with the left hand. In addition, there was mild dysmetria on hand to chin testing bilaterally, minimal left side cogwheel rigidity and some difficulty with tandem gait. Finally, there was mild decrease in pinprick sensation in both feet. The rest of the examination was normal, with specifically no bradykinesia or shuffling gait.

The patient was diagnosed with essential tremor (ET). The presence of cogwheel rigidity would not exclude the diagnosis of ET. However, because of the onset of tremor later in life and the low score on the MOCA, testing for FXTAS premutation was ordered. In addition, a brain MRI, neurocognitive testing, as well as serum levels of TSH and ceruloplasmin were ordered. Metoprolol was increased to $100 \mathrm{mg}$ daily for symptomatic control.

The brain MRI was normal, as were the TSH, ceruloplasmin and electrolytes levels. The neuropsychological evaluation was not diagnostic for dementia but revealed borderline IQ with poor verbal skills (naming, vocabulary, verbal reasoning), mild frontal dysfunction and low average memory, attention, visuospatial skills and processing speed. However, the FMR1 genetic testing revealed 2 alleles, one with 20 and the other with 30 CGG repeats. While the number of repeat was in the normal range $(<55)$ and not diagnostic of FXTAS, the presence of 2 alleles suggested a chromosomal abnormality as this male patient would be expected to have only one chromosome X. These results were suspicious of KS, which was confirmed by karyotyping.

\section{Discussion}

While KS is the most common aneuploidy in men, affecting $0.1-0.2 \%$ of the general population [1], only 2 patients reported in the literature have previously been diagnosed with KS while being tested for FXTAS $[3,4]$. 
Page 2 of 2

Harlow et al. [3] reported a 68-year-old man with a 5 year history of bilateral upper extremity action tremor that subsequently involved his head and his voice. He had also complained of poor balance and memory decline for a year. He had no biological children.

The combination of action tremor, ataxia and cognitive decline prompted genetic testing for FXTAS. The CGG repeat number was normal but two alleles were found, prompting an evaluation for Klinefelter syndrome.

Continho et al. [4] reported the case of a 56 year old male with a 3 years history of bilateral hand and postural action tremor, as well as learning difficulties since childhood with more recent cognitive decline, and inability to conceive. A neurocognitive assessment reported below average results in multiple areas, particularly language and executive functions. Because of the co-existence of tremor and cognitive decline, genetic testing for FXTAS was ordered. Similarly, the CGG repeat number was normal but two alleles were found, prompting an evaluation for Klinefelter syndrome.

These cases are strikingly similar to ours: older male with postural and action tremor in the hands and cognitive impairment, unable to conceive, suspected of having FXTAS, but diagnosed with KS after they were found to have 2 FMR1 alleles. However, the patient of Harlow et al did not have lifelong learning difficulty, while ours and Continho's did. In addition, ataxia was marked in Harlow's patient, minimal in ours and seemingly absent in Continho's.

The suspicion of gabapentin-induced ataxia could be raised in our patient. However, there was no subjective change in his gait and ataxia on examination was minimal, which contrasts with the more severe gabapentin induced ataxia.

In a controlled study on 44 patients with Klinefelter syndrome (KS) and 95 controls, Harlow and Gonzalez-Alegre [2] reported that postural and action tremor was 5 to 10 times more frequent in the KS population compared to the general population. That tremor would rarely affect the voice or the head. In addition, KS patients tended to develop tremor earlier $(20 \mathrm{v} / \mathrm{s} 38$ years of age, $\mathrm{p}=0.012)$ and to report poor balance. However the 3 patients we discuss here developed tremor after the age of 50. While Harlow and Gonzalez-Alegre's study confirmed previous uncontrolled reports that KS patients have a high prevalence of postural tremor, it suggested correcting the phenomenology from ET-like tremor to FXTAS -like symptomatology because of the higher incidence of ataxia in KS patients [2,5].

While cognitive decline and tremor can also be observed in patients suffering from some neurodegenerative diseases, such as Alzheimer's disease [6], these typically do not report difficulty conceiving. On the other hand, infertility is a constant complaint in KS [7], and its presence in addition to cognitive decline and tremor should raise the suspicion of KS rather than dementia.

The pathophysiology of tremor in KS patients is unclear. It has been suggested that, since cortical and subcortical oscillators are involved [8], damage to the corticothalamic fibers could account for it [4]. This could be secondary to abnormal brain development and function, either directly from the chromosomal defect [9], or indirectly through hormonal dysregulation on the developing brain [2]. A direct effect of hormonal dysregulation on the adult brain is less likely as tremor tends to be resistant to hormonal therapy [3].

Our case adds to only 2 similar patients previously reported, in whom tremor and cognitive decline prompted genetic testing for FXTAS, which result suggested KS.

We here intend to draw attention to Klinefelter syndrome as the cause of not only tremor, but also imbalance, and to underline the need to suspect it in the presence of tremor and cognitive decline, especially when a patient has history of infertility.

\section{References}

1. Lanfranco F, Kamischke A, Zitzmann M, Nieschlag E (2004) Klinefelter's syndrome. Lancet 364: 273-283.

2. Harlow TL, Gonzalez-Alegre P (2009) High prevalence of reported tremor in Klinefelter syndrome. Parkinsonism Relat Disord 15: 393-395.

3. Harlow TL, Rodnitzky RL, Gonzalez-Alegre P (2008) From essential tremor to Klinefelter through Fragile X, an unexpected journey. Mov Disord 23: 1328-1329.

4. Coutinho E, Pereira JP, Dias D, Cavaco S, Pinto C, et al. (2010) Tremor and white matter lesions in Klinefelter syndrome. Mov Disord 25: 1993-1994.

5. Hecht A, Ruskin H (1960) Seminiferous tubule dysgenesis (Klinefelter's syndrome) associated with familiar cerebellar ataxia. J Clin Endocrinol Metab 20: 1184-1190.

6. LaRoia H, Louis ED (2011) Association between essential tremor and other neurodegenerative diseases: what is the epidemiological evidence? Neuroepidemiology 37: 1-10.

7. Dávila Garza SA, Patrizio P (2013) Reproductive outcomes in patients with male infertility because of Klinefelter's syndrome, Kartagener's syndrome, round-head sperm, dysplasia fibrous sheath, and 'stump' tail sperm: an updated literature review. Curr Opin Obstet Gynecol 25: 229-46.

8. Raethjen J, Deuschl G (2009) Tremor. Curr Opin Neurol 22: 400-405.

9. Deng H, Le W, Jankovic J (2007) Genetics of essential tremor. Brain 130: 1456-1464. 